\title{
Biological Serum Markers in the Management of Pediatric Pulmonary Arterial Hypertension
}

\author{
MIRJAM E. VAN ALBADA, FREDERIEKE G. LOOT, REBECCA FOKKEMA, MARCUS T. R. ROOFTHOOFT, AND \\ ROLF M. F. BERGER
}

\begin{abstract}
Department of Pediatrics, Division of Pediatric Cardiology [M.E.A., F.G.L., M.T.R.R., R.M.F.B.] and Department of Pathology and Laboratory Medicine [R.F.], Beatrix Children's Hospital/University Medical Center Groningen, University of Groningen, 9700 RB Groningen, The Netherlands
\end{abstract}

\begin{abstract}
Appropriate parameters are needed for the monitoring of children with pulmonary arterial hypertension (PAH). Various biologic markers seem to be of use in adults with PAH. No data are available on their value in children with PAH. In this study, the relation between serum markers, functional parameters, and hemodynamic variables in pediatric $\mathrm{PAH}$ and their ability to predict survival is determined. Serum N-terminal pro brain natriuretic peptide (NT-proBNP), uric acid, norepinephrine, and epinephrine were measured and correlated with invasive hemodynamics, functional parameters, and outcome in 29 pediatric patients with PAH who visited a tertiary reference center for pediatric PAH between 1997 and 2005. NT-proBNP correlated with functional class $(R=0.36$; $p=0.03)$ and 6-min walking distance (6MWD) $(R=-0.53 ; p<$ $0.001)$. Uric acid correlated with mean pulmonary arterial pressure, pulmonary vascular resistance, and cardiac index $(R=0.63, p=$ $0.01 ; R=0.71, p=0.03$, and $R=-0.65, p=0.007$, respectively). After initiation of treatment, NT-proBNP decreased. This decrease correlated with an increased 6MWD. Finally, norepinephrine and NT-proBNP levels were highly predictive for mortality. In this series of children with PAH, biologic markers were correlated with hemodynamics and functional capacity, as parameters of disease severity. The data indicate that these markers can be used to monitor treatment effects and predict mortality in pediatric PAH. (Pediatr Res 63: 321-327, 2008)
\end{abstract}

P ulmonary arterial hypertension (PAH) is a chronic, progressive, and usually fatal disease. It is characterized by proliferation of pulmonary vascular cells and obliteration of small pulmonary arteries, leading to increased pulmonary vascular resistance and eventually right heart failure and death. PAH may present at any age, from infancy into high age. In the last decade, new pharmacological therapies have been developed that improve hemodynamics, exercise capacity, and survival in these patients. For optimal clinical decision-making, it has become of growing importance to accurately assess disease severity, effectiveness of therapy, and prognosis in the individual patient with PAH.

Several correlates of disease severity and survival have been described in adults with PAH. Hemodynamic and functional capacity parameters are currently the cornerstones in

Received July 25, 2007; accepted November 3, 2007.

Correspondence: Rolf M. F. Berger, M.D., Ph.D., Pediatric Cardiology, Beatrix Children's Hospital, University Medical Center Groningen, Postbus 30.001, 9700 RB Groningen, The Netherlands; e-mail: r.m.f.berger@bkk.umcg.nl characterizing disease progression. Invasively obtainable hemodynamic parameters have been shown to represent disease severity and to predict survival (1). Noninvasive parameters for functional capacity are used for the assessment of clinical condition, severity of disease, and effectiveness of therapy. Six-min walking distance (6MWD) has been shown to correlate well with World Health Organization (WHO) functional class, and also with hemodynamic parameters (2). Further, maximal cardiopulmonary exercise testing can be safely performed in these patients using specific guidelines, and is used with increasing frequency (3).

In pediatric patients with $\mathrm{PAH}$, the use of these parameters has specific drawbacks. As in adults, cardiac catheterization is invasive and is associated with specific risks. In the pediatric age group, however, cardiac catheterization is mostly performed under general anesthesia, making repetitive catheterizations for follow-up unattractive. Exercise capacity tests as the 6MWD or maximal exercise tests are often not feasible and less validated in young childhood (4). Therefore, additional parameters to monitor disease severity, prognosis, and efficacy of treatment are highly needed in pediatric patients with PAH.

In PAH, the neurohumoral axis is activated, as evidenced by elevated circulating levels of brain natriuretic peptide (BNP), $\mathrm{N}$-terminal pro brain natriuretic peptide (NT-proBNP), catecholamines, and other neurohumoral markers (5-9). Also, uric acid levels have shown to be increased in adults with PAH, both idiopathic and secondary (10). Studies in adult patients with PAH have suggested that NT-proBNP (7), norepinephrine (9), and uric acid (11) are correlated with hemodynamic and functional parameters and could be used for monitoring therapy effects and prognosis in these patients. Although appropriate reference values are lacking in children, preliminary data suggest that BNP and NT-proBNP levels are useful in diagnosing and managing pediatric heart failure, congenital heart disease, and cardiac transplantation (12).

In this study, we aimed to investigate the value of uric acid, NT-proBNP, epinephrine, and norepinephrine in a cohort of

Abbreviations: 6MWD, six-minute walking distance; BNP, brain natriuretic peptide; GFR, glomerular filtration rate; NT-proBNP, N-terminal pro brain natriuretic peptide; PAH, pulmonary arterial hypertension; WHO, World Health Organization 
children with $\mathrm{PAH}$, with respect to predicting prognosis and monitoring disease severity and effectiveness of therapy as assessed by hemodynamic and functional parameters.

\section{METHODS}

Patients. Twenty-nine consecutive pediatric patients in whom PAH was diagnosed in a tertiary reference center for pediatric pulmonary hypertension between 1997 and 2005 were followed using a standardized protocol, in which WHO functional class, physical examination, blood withdrawal for biologic markers, and a 6-min walking test were performed every 3-6 mo. The study was approved by the Institutional Review Board. Informed consent was obtained from the parents of all children.

Cardiac catheterization. Cardiac catheterization was performed under general anesthesia at presentation to confirm the diagnosis PAH. In 16 patients, concomitant blood samples were collected before the catheterization procedure. Complete hemodynamic data were obtained, including systemic and pulmonary blood flows using the Fick method and vascular resistances, indexed for body surface.

Blood sampling and assays. Peripheral venous blood samples were further obtained at each outpatient visit. For prognostic value, serum uric acid was determined at presentation, whereas the other serum markers were collected from 2003, when the role of the neurohumoral axis in pulmonary hypertension became more obvious. Blood samples drawn at start of new therapy (either as first line or add-on therapy) and 3 mo later were used to determine the value of the different serum markers in the assessment of therapeutic efficacy.

To determine NT-proBNP, blood was collected in EDTA tubes, transported on ice and stored at $-20^{\circ} \mathrm{C}$. Plasma NT-proBNP was determined with an Elecsys ${ }^{\circledR}$ proBNP assay (Roche Diagnostics, Basel, Switzerland). Uric acid and serum creatinine were measured using standard clinical chemical methods with a MEGA (Merck, Darmstadt, Germany). For determination of catecholamines, samples were stored at $-20^{\circ} \mathrm{C}$ in glass tubes containing glutathione solution and measured using chromatography. All blood samples were drawn after a 10-min stabilization period of the patient in a horizontal position.

Statistical analysis. All data are expressed as mean value \pm SEM unless otherwise indicated. Log transformation was used to normalize the distribution of variables. Correlations between variables were measured using Pearson's correlation coefficient or, in case of noncontinuous variables, Spearman's $\rho$ correlation coefficient. To analyze the relation between the two functional parameters WHO-class and 6MWD and simultaneously derived serum markers, we included the first three visits per patient to increase sample size, leaving 20 patients and 60 data points. The relation between WHO-class and serum markers was obtained by logistic regression analysis, after transformation of the classification scores into a dichotomous variable (either $<3$ or $\geq 3$ ). An estimated glomerular filtration rate (GFR) was calculated for each patient at the time of cardiac catheterization using the formula $(38 \times$ height $(\mathrm{cm})) /$ creatinine $(\mu \mathrm{mol} / \mathrm{L})$ and the relation between hemodynamics and uric acid was corrected for GFR and diuretic use in a multivariate regression analysis. Multivariate regression analysis was further used to correct for diagnosis, discriminating between idiopathic, and secondary forms of PAH.

To determine the value of the biologic markers in relation to effectiveness of therapy, serum levels at the start of treatment were compared with those 3 mo later with a paired sample $t$ test or a Wilcoxon signed ranks test when data were not normally distributed.

For Kaplan-Meier survival curves, patients were divided into high, middle, and low categories, with an equal number of patients in each group, using a log-rank test based on trends. Cox regression analyses were performed to be able to correct for diagnosis group. Receiver operating characteristics were generated from multiple sensitivity/specificity pairs. All analyses were performed using SPSS $\odot$ version 12.02 for Windows (SPSS Inc., Chicago, IL). A $p$-value $<0.05$ was considered to be significant.

\section{RESULTS}

Twenty-nine patients were included in this study. Eighteen children were diagnosed with idiopathic $\mathrm{PAH}$ and 11 patients with PAH due to a congenital left to right shunt. Of these, 10 had Eisenmenger syndrome with a posttricuspid defect: patent arterial duct (PDA) $n=2$, ventricular septal defect (VSD) $n=$ 7 (4 of which combined with a PDA), AVSD $n=1$. One patient had a surgically corrected truncus arteriosus. Median age at inclusion was $7.0 \mathrm{y}$ (range, 0.1-17.3). Girls were slightly older than boys [median age in girls 7.8 (2.9-17.3) and in boys $4.0(0.1-15.2), p=0.06]$. Median and range of all serum markers at the first sample collection for survival analysis was as follows: NT-proBNP 138 (27-7589) pg/mL, uric acid $0.29(0.12-0.56) \mathrm{mmol} / \mathrm{L}$, norepinephrine 1.46 (0.39-9.60) nmol/L, epinephrine $0.19(0.03-1.68) \mathrm{nmol} / \mathrm{L}$. Two patients were not included in the survival analysis due to incomplete data. The characteristics of the patients included in the survival analysis are provided in Table 1.

Patients were treated as clinically indicated. At the time of inclusion, 19 patients received anticoagulants, 9 patients received diuretics, 7 patients received a calcium-antagonist, 1 patient received digoxin, 6 patients received bosentan, 2 patients received epoprostenol, and 1 patient received beraprost sodium. During follow-up, 6 patients were started on anticoagulants, 2 on diuretics, 1 on digoxin, and 14 patients were started on bosentan; 4 patients were started on epoprostenol and 1 patient received treatment with sildenafil.

Median follow up was 30 mo (range, 4-156 mo). During this follow-up, 9 patients (31\%) died because of circulatory insufficiency due to progressive right ventricular failure, one of these during massive hemoptysis.

Demographic data. Uric acid was correlated with age $(R=$ $0.431, p=0.03$ ) and sex (males $0.25 \pm 0.02 \mathrm{mmol} / \mathrm{L}$ versus females $0.32 \pm 0.03 \mathrm{mmol} / \mathrm{L}, p=0.04$ ). Uric acid did not differ between patients with idiopathic or secondary PAH (idiopathic $0.28 \pm 0.03 \mathrm{mmol} / \mathrm{L}$ versus secondary $0.32 \pm 0.03$ $\mathrm{mmol} / \mathrm{L}, p=0.36$ ). NT-proBNP, norepinephrine, and epinephrine did not correlate with sex, age, or diagnosis.

Hemodynamics. In 16 patients, concomitant biologic markers and invasive hemodynamic data were available. Serum uric acid correlated with mean pulmonary arterial pressure $(R=$ $0.63, p=0.01$, Fig. $1 A)$ and pulmonary vascular resistance $(R=$ $0.71, p=0.03$, Fig. $1 B)$. A negative relation with cardiac index was demonstrated $(R=-0.65, p=0.007$, Fig. $1 C)$. After correction for GFR, these correlations remained basically unchanged $(R=0.66, p=0.08 ; R=0.69, p=0.03$; and $R=$ $-0.65, p=0.04$, respectively). In addition, after correction for diuretic use or diagnosis group, the correlations remained unchanged (correction for diuretic use $R=0.67, p=0.03 ; R=$ $0.71, p=0.02$; and $R=-0.71, p=0.01$, respectively and correction for diagnosis group $R=0.67, p=0.03 ; R=0.74, p=$ 0.01 ; and $R=-0.73, p=0.007$, respectively). Further, a positive correlation between right atrial pressure and norepinephrine was suggested $(R=0.62, p=0.06)$. NT-proBNP and epinephrine levels did not correlate with hemodynamic variables.

Functional capacity. A higher WHO classification was associated with a decreased 6MWD (logistic regression $R=$ $-0.58, p=0.001)$. NT-proBNP correlated with $6 \mathrm{MWD}$ as tested on the day of blood sampling $(R=-0.527, p<0.001$, Fig. 2A). A higher NT-proBNP was also associated with higher WHO-classification (logistic regression $R=0.34, p=$ 0.04 ; Fig. $2 B$ ). These correlations were independent from diagnosis group $(R=-0.57, p<0.001$ and $R=0.55, p=$ 0.002 , respectively). No correlations could be demonstrated between the other biologic markers and these functional parameters. 
Table 1. Patient characteristics of patients in survival analysis

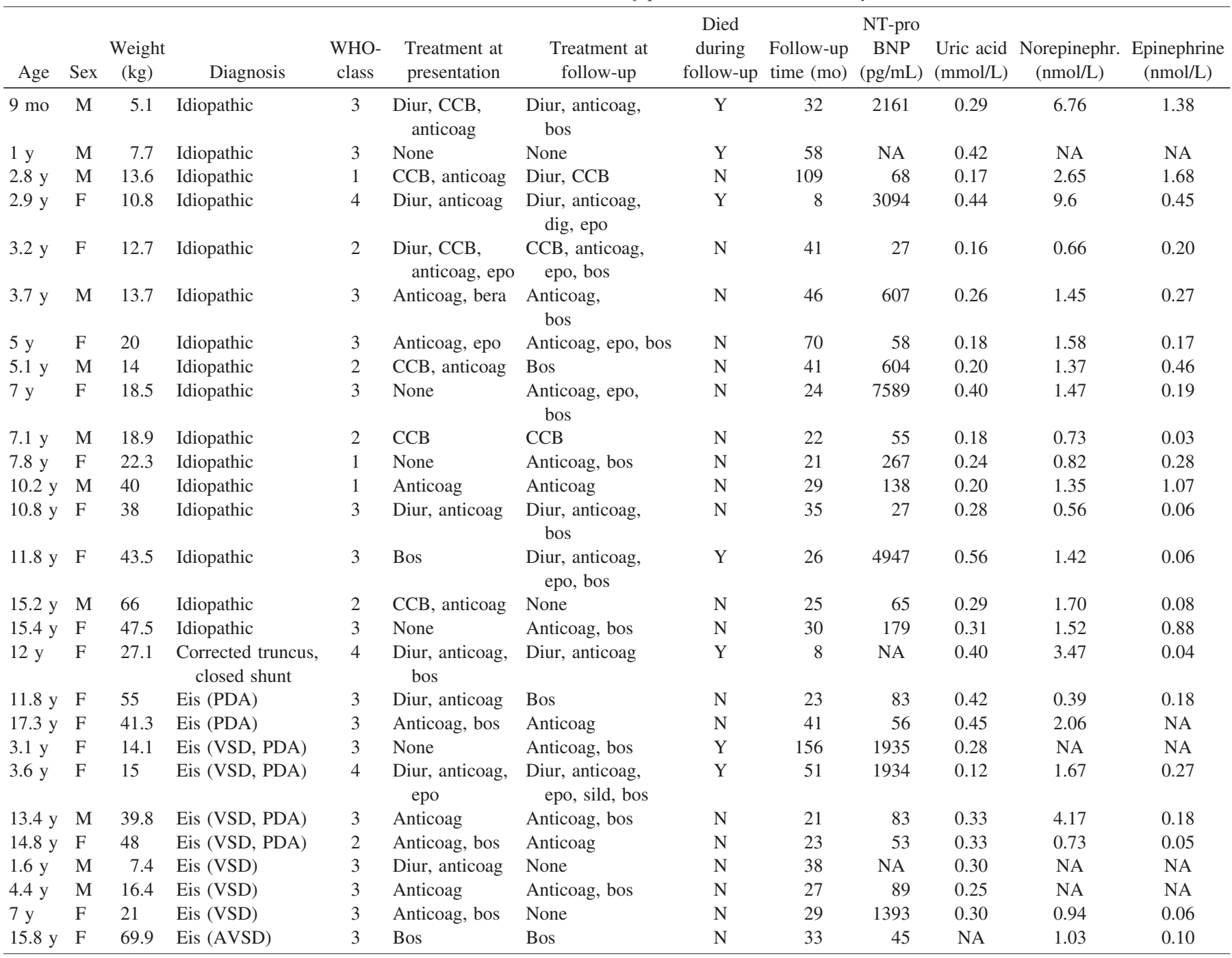

M, male; F, female; mo, months; y, years; Eis, Eisenmenger; VSD, ventricular septal defect; PDA, patent ductus arteriosus; AVSD, atrioventricular septal defect; diur, diuretics; CCB, calcium-channel blocker; anticoag, anticoagulant; epo, epoprostenol; bera, beraprost; sild, sildenafil; bos, bosentan; NA, not available.
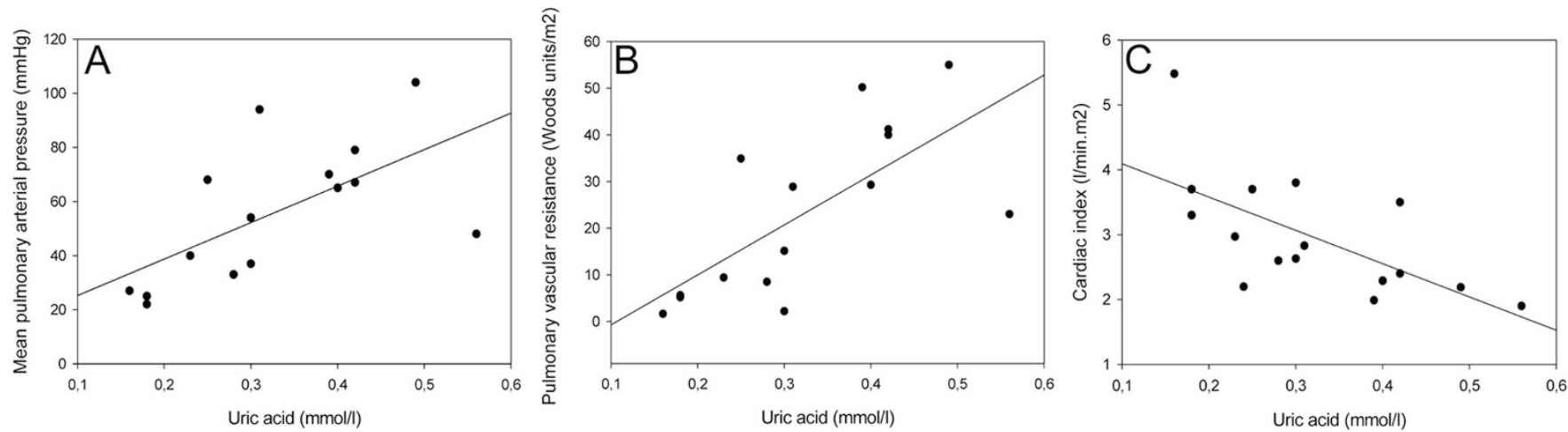

Figure 1. Relation between serum uric acid and $(A)$ mean pulmonary arterial pressure $(R=0.63, p=0.01),(B)$ pulmonary vascular resistance $(R=0.71, p=$ $0.03)$, and $(C)$ cardiac index $(R=0.65, p=0.007)$.

Treatment effect. In 13 patients, biologic markers, WHOclass and 6MWD were compared at initiation of therapy with either bosentan $(n=10)$, epoprostenol $(n=2)$, or sildenafil $(n=1)$ and 3 mo later. NT-proBNP levels were significantly decreased after 3 mo of therapy compared with levels before therapy (mean \pm SEM $631 \pm 228 \mathrm{pg} / \mathrm{mL}$ versus $1484 \pm 600$ $\mathrm{pg} / \mathrm{mL}$, respectively, $p=0.016$ ). In these patients, $6 \mathrm{MWD}$ increased more than $50 \mathrm{~m}(302 \pm 32 \mathrm{~m}$ before therapy versus $367 \pm 30$ after 3 mo of treatment, $p=0.03$ ) and WHO-class improved $(3.0 \pm 0.1$ before therapy versus $2.5 \pm 0.1$ after 3 

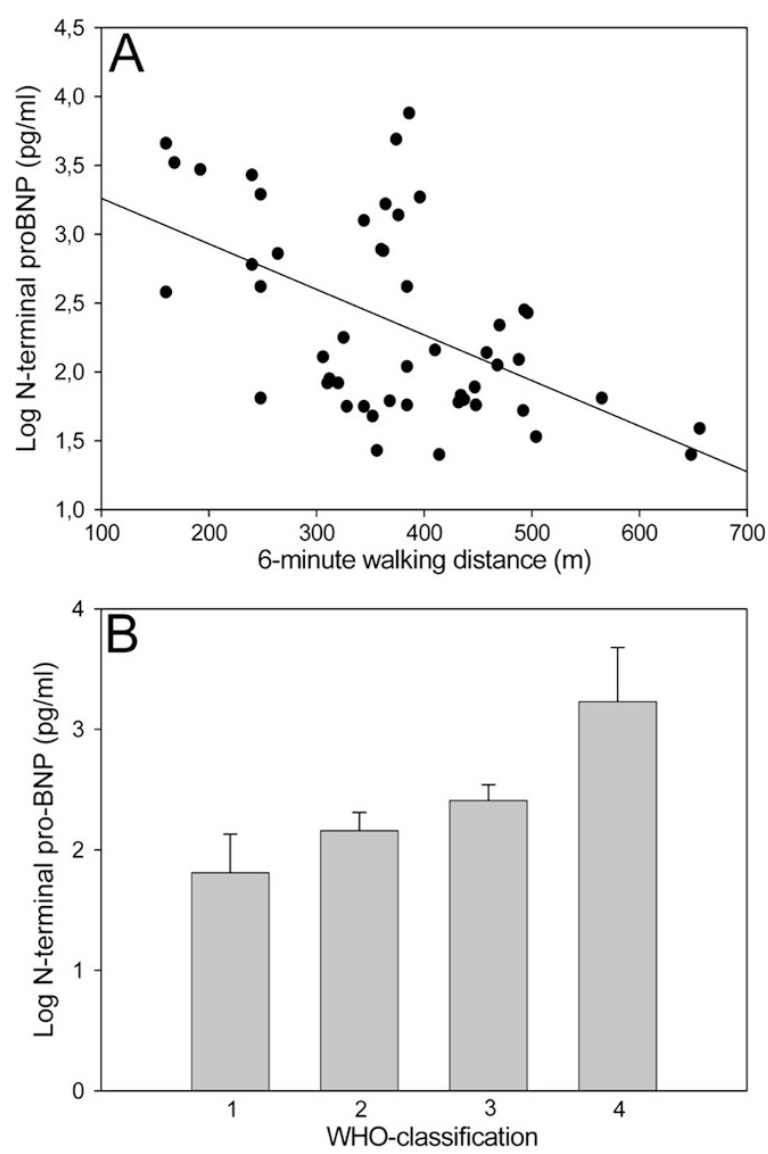

Figure 2. Relation between serum markers and functional parameters: $(A)$ $\log$ N-terminal pro-BNP and 6MWD ( $n=3$ per patient in 20 patients). $(B) \log$ N-terminal pro-BNP and WHO-classification $(n=20)$.

mo of treatment, $p=0.006$ ). The size of the change in NT-proBNP was related to the size of the change in $6 \mathrm{MWD}$ $(R=-0.63, p=0.04)$ and in WHO class $(R=0.72, p=$ $0.02)$. No changes in the levels of uric acid, norepinephrine, and epinephrine could be demonstrated after 3 mo of treatment.

Prognosis. Of the 29 patients studied, 9 (31\%) died during follow-up, 3 boys and 6 girls. The Kaplan-Meier survival curves of the different serum markers are shown in Fig. 3. High NT-proBNP, uric acid, and norepinephrine levels all predicted increased mortality (Cox-regression: $\chi^{2}=9.93, p=$ $0.002 ; \chi^{2}=5.93, p=0.015$; and $\chi^{2}=15.39, p<0.001$, respectively), independently of diagnosis (Cox-regression with diagnosis as covariate: $\chi^{2}=10.79, p=0.005 ; \chi^{2}=6.29$, $p=0.043$; and $\chi^{2}=15.56, p<0.001$, respectively). ROCcurves for NT-proBNP, uric acid, and norepinephrine predicting mortality are provided in Fig. 4. NT-proBNP and norepinephrine both had an area under the curve $>0.85$. When using a cutoff value of $1664 \mathrm{pg} / \mathrm{mL}$ for NT-proBNP, the test would have a $100 \%$ sensitivity and a $94 \%$ specificity in predicting mortality. Norepinephrine with a cutoff of $1.63 \mathrm{nmol} / \mathrm{L}$ has a $80 \%$ sensitivity and $77 \%$ specificity in predicting mortality. The area under the ROC curve for uric acid was 0.65 and did not differ significantly from 0.5 .

\section{DISCUSSION}

This study demonstrates that serum levels of NT-proBNP, uric acid, and norepinephrine can be used in the assessment of disease severity, prognosis, and effectiveness of therapy in children with PAH.

Uric acid serum levels correlated with invasively obtained hemodynamic data, whereas NT-proBNP levels correlated with functional outcome parameters as WHO-class and 6MWD. NT-proBNP levels decreased after initiation of therapy and this decrease correlated with the functional response to the initiation of this treatment. Furthermore, serum levels of NT-proBNP and norepinephrine were highly predictive for mortality in the individual patient.

Neurohumoral axis activation occurs in different types of cardiovascular conditions, including heart failure and congenital heart disease and is evidenced by increased serum levels of markers such as epinephrine, norepinephrine, and NT-proBNP. Therefore, it has been suggested that serum levels of neurohormones may function as biologic markers for disease progression and prognosis and may be useful in the detection and diagnosis of heart failure and in predicting morbidity and mortality in several cardiovascular conditions (13-17). Furthermore, an increased serum level of uric acid is associated with increased morbidity and mortality in heart failure and congenital heart disease (18-21). Increased serum uric acid in heart failure is most probably caused by increased activity of
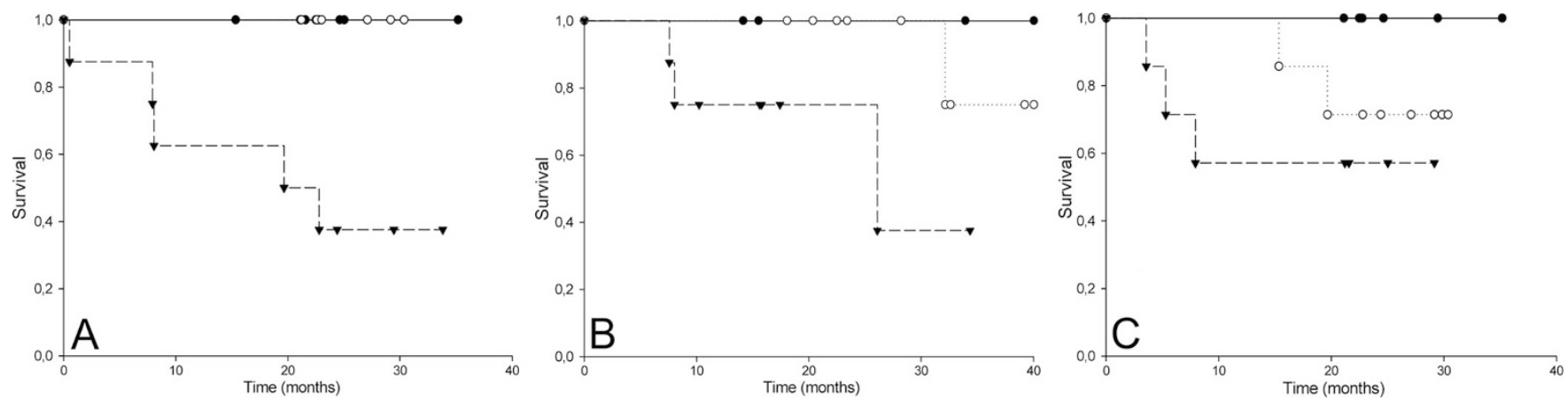

Figure 3. Kaplan-Meier survival curves for the different biochemical markers: Cumulative survival estimated by Kaplan-Meier curves for $(A)$ NT-proBNP, -- -- indicates NT-pro-BN $p \leq 80 \mathrm{pg} / \mathrm{mL}, \cdots \circ \cdots$ indicates NT-pro-BNP between 80 and $605 \mathrm{pg} / \mathrm{mL}$, and $--\nabla--$ indicates NT-pro-BNp $\geq 605 \mathrm{pg} / \mathrm{mL}, p=$ 0.004; $(B)$ for uric acid, -- -- indicates uric acid $\leq 0.25 \mathrm{mmol} / \mathrm{L}, \cdots \circ \cdots$ indicates uric acid between 0.25 and $0.32 \mathrm{mmol} / \mathrm{L}$, and $--\nabla--$ indicates uric acid $\geq 0.32$ $\mathrm{mmol} / \mathrm{L}, p=0.01 ;(C)$ for norepinephrine, $--\bullet--$ indicates norepinephrine $\leq 1.00 \mathrm{nmol} / \mathrm{L}, \cdots 0 \cdots$ indicates norepinephrine between 1.00 and $1.68 \mathrm{nmol} / \mathrm{L}$, and - $\boldsymbol{\nabla}-$-indicates norepinephrine $\geq 1.68 \mathrm{nmol} / \mathrm{L}, p=0.04$. $p$-values are derived from over all log-rank testing for trends. 

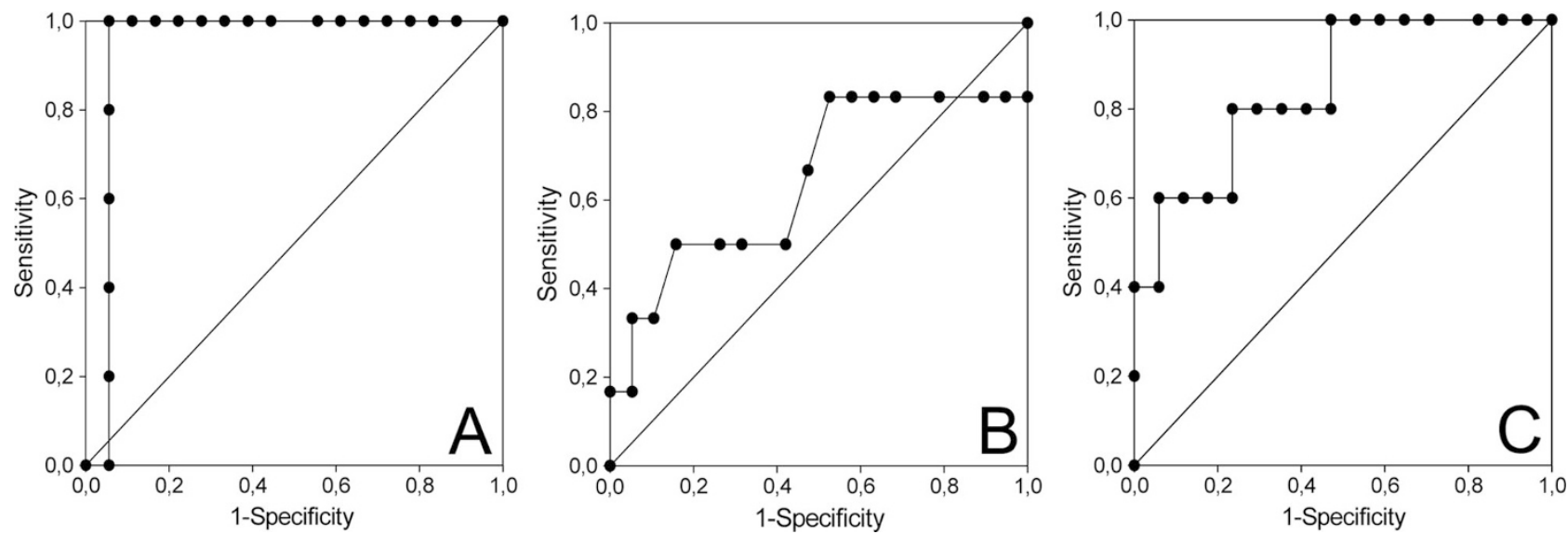

Figure 4. ROC-analysis for serum NT-proBNP $(A ; \mathrm{AUC}=0.94, p=0.003)$, uric acid $(B ; \mathrm{AUC}=0.65, p=0.294)$, and norepinephrine $(C$; AUC $=0.85$, $p=0.02)$ in the prediction of death in pediatric patients with PAH. AUC $=$ area under the curve.

the enzyme xanthine oxidase. Xanthine oxidase catalyzes the oxidation of xanthine to uric acid. In patients with heart failure, inhibition of xanthine oxidase activity with allopurinol improved vascular function (22), indicating that xanthine oxidase activity is directly contributing to the pathogenetic process in heart failure patients. Because uric acid has been shown to be increased in pulmonary hypertensive patients (11), xanthine oxidase activity may also be a mediator in the pathobiological mechanisms of pulmonary hypertension.

Disease severity in PAH is characterized by hemodynamics and functional capacity.

Hemodynamic parameters, including right atrial pressure, pulmonary arterial pressure, pulmonary vascular resistance, and cardiac index, are considered indicators for the severity of disease in PAH and are associated with prognosis $(1,23,24)$. In the present study, of the biologic markers investigated, uric acid level displayed the strongest correlations with invasive hemodynamics. It correlated with mean pulmonary arterial pressure, pulmonary vascular resistance and cardiac index, but not with right atrial pressure. To exclude the possibility that hyperuricemia was caused by impaired kidney function or diuretic use, a multivariate analysis was performed to correct for these variables. However, the correlations appeared to be independent of these factors in our pediatric series. Further, relations were identical in patients with either idiopathic $\mathrm{PAH}$ or PAH associated with congenital heart defects. In adult PAH patients, uric acid has been correlated with right atrial pressure $(10,25)$, pulmonary vascular resistance (11), and cardiac index $(11,25)$. Norepinephrine levels have also been described to correlate with hemodynamic parameters in adult PAH, although reported data are not conclusive. Nootens et al. (9) found a significant correlation between norepinephrine levels and pulmonary arterial pressures, pulmonary vascular resistance and cardiac index in 21 adult patients with idiopathic pulmonary hypertension, whereas Nagaya et al. (6) could only demonstrate a correlation with cardiac index. In the current pediatric study, we found that norepinephrine tended to correlate with right atrial pressure, but not with other hemodynamic parameters.

No correlations between serum levels of NT-proBNP and hemodynamic parameters could be demonstrated in the cur- rent study. This is in contrast with studies in adult patients with PAH and systemic sclerosis, in which NT-proBNP was found to correlate with pulmonary arterial pressure $(26,27)$. Similar to our data, Fijalkowska et al. could not demonstrate a relation with pulmonary arterial pressure in patients with idiopathic PAH (7).

Correlations of NT-proBNP levels with right atrial pressure, pulmonary vascular resistance and cardiac index have been described in adults with PAH $(7,27,28)$.

Functional capacity. We found NT-proBNP to correlate well with the functional status of pediatric PAH patients, characterized by WHO-functional class and 6MWD, independent of diagnosis group. This is in concordance with findings in adult patients, in whom also correlations with these parameters have been described $(7,27,28)$. At present, 6MWD is generally accepted as a valuable parameter for clinical status and a predictor for outcome in PAH patients. In pediatric patients, the 6MWD is not always feasible because of young age or lack of cooperation. Furthermore, 6MWD has not been validated in children younger than 8 y of age. Our findings indicate that NT-proBNP serum levels may form a substitute for the 6MWD in pediatric patients with PAH. Serum levels of uric acid and norepinephrine did not correlate with parameters of functional class in our study. In contrast, both uric acid and norepinephrine have been reported to be correlated with WHO functional class in adult patients with PAH $(9,11)$.

Increased levels of NT-proBNP have been associated with poor long-term prognosis in adults with pulmonary hypertension. Fijalkowska et al. reported that a serum NT-proBNP higher than $1400 \mathrm{pg} / \mathrm{mL}$ identified patients with a poor longterm prognosis with a $88 \%$ specificity and a $53 \%$ specificity (7). In this pediatric study, 5 of 6 patients $(83 \%)$ with a NT-proBNP level higher than this level died within $2 \mathrm{y}$, while all patients with a level below $1400 \mathrm{pg} / \mathrm{mL}$ survived during follow-up. When we used a cutoff value of $1664 \mathrm{pg} / \mathrm{mL}$, sensitivity and specificity for predicting mortality could even be improved to $100 \%$ and $94 \%$, respectively. In other words, in this study NT-proBNP showed to be an excellent predictor of mortality that can be used in the individual pediatric patient with PAH. 
Norepinephrine serum level was also a predictor for mortality in our study. Although not as strong as NT-proBNP, the area under the ROC curve was 0.85 . These findings are in congruency with data in adult patients with iPAH as reported by Nagaya et al. (6). In contrast, no correlation could be demonstrated between epinephrine levels and mortality. This also is in congruency with findings in adults with PAH (8).

Further, uric acid serum level has been described as a prognostic factor in adult PAH (29). In the current pediatric series, uric acid levels also appeared to be correlated with mortality. However, the ROC-curve showed that uric acid level was less valuable in predicting mortality in the individual child. It should be noted that, in the current study, the different biologic markers predicted mortality, irrespective of the received treatment.

Additionally, our study suggests that NT-proBNP can be useful in monitoring treatment effects in pediatric patients with PAH. Treatment was associated with a decrease in serum NT-proBNP levels and this decrease correlated with improvement in 6MWD. The number of patients and observations in this study did not allow answering the question if the magnitude of changes in NT-proBNP serum levels after initiation of therapy was predictive for outcome or affected the value of baseline NT-proBNP level in predicting mortality in the individual patient. Our data indicate that NT-proBNP may be used to evaluate the effect of treatment in children with PAH. This is of especially great importance in the youngest patients in whom the accepted clinical endpoints applied for PAH are often not feasible to obtain.

No healthy control group was used in this study. Normal values for serum levels of the described biologic markers in children are not fully established. Therefore, the value of biologic markers in diagnosing the presence of PAH in children could not be determined in this study. However, the value of these markers as correlates for disease severity, prognosis, and treatment effect in the specific condition of pediatric PAH could be established.

The 6-min walking test has not been validated in children younger than $8 \mathrm{y}$ of age. Lack of cooperation at younger age may hamper its results. Seven of our patients underwent at least one 6-min walking test between 5 and $7 \mathrm{y}$ of age. In our experience, familiarizing the young child with the test by training can result in reproducible 6MWD assessments from the age of $5 \mathrm{y}$.

In conclusion, this study demonstrates that serum levels of NT-proBNP, uric acid, and norepinephrine can be used for the assessment of disease severity, prognosis, and effectiveness of therapy in children with PAH. These biologic serum markers may therefore allow to support clinical decision making in the management of the individual child with $\mathrm{PAH}$, especially in those in whom obtaining accepted endpoint parameters is not feasible (exercise capacity tests) or unattractive (repeated invasive hemodynamic evaluation). Further prospective studies are warranted, to assess the predictive value of treatment induced changes in the serum levels of these markers.

Acknowledgments. The Elecsys ${ }^{\circledR}$ proBNP assay was generously provided by Roche Diagnostics, Basel, Switzerland.

\section{REFERENCES}

1. D'Alonzo GE, Barst RJ, Ayres SM, Bergofsky EH, Brundage BH, Detre KM, Fishman AP, Goldring RM, Groves BM, Kernis JT 1991 Survival in patients with primary pulmonary hypertension. Results from a national prospective registry. Ann Intern Med 115:343-349

2. Miyamoto S, Nagaya N, Satoh T, Kyotani S, Sakamaki F, Fujita M, Nakanishi N, Miyatake K 2000 Clinical correlates and prognostic significance of six-minute walk test in patients with primary pulmonary hypertension. Comparison with cardiopulmonary exercise testing. Am J Respir Crit Care Med 161:487-492

3. Wensel R, Opitz CF, Anker SD, Winkler J, Hoffken G, Kleber FX, Sharma R, Hummel M, Hetzer R, Ewert R 2002 Assessment of survival in patients with primary pulmonary hypertension: importance of cardiopulmonary exercise testing. Circulation 106:319-324

4. Ivy DD 2006 Pulmonary arterial hypertension assessment in pediatric cardiology. In: Beghetti M, Barst RJ, Naeije R, Rubin LJ (eds) Pulmonary Arterial Hypertension Related to Congenital Heart Disease. Elsevier GmbH. Munich, Germany, pp 93-111

5. Tunaoglu FS, Olgunturk FR, Gokcora N, Turkyilmaz C, Gurbuz F 1994 Atrial natriuretic peptide concentrations in children with pulmonary hypertension: correlation with hemodynamic measurements. Pediatr Cardiol 15:288-295

6. Nagaya N, Nishikimi T, Uematsu M, Satoh T, Kyotani S, Sakamaki F, Kakishita M, Fukushima K, Okano Y, Nakanishi N, Miyatake K, Kangawa K 2000 Plasma brain natriuretic peptide as a prognostic indicator in patients with primary pulmonary hypertension. Circulation 102:865-870

7. Fijalkowska A, Kurzyna M, Torbicki A, Szewczyk G, Florczyk M, Pruszczyk P, Szturmowicz M 2006 Serum N-terminal brain natriuretic Peptide as a prognostic parameter in patients with pulmonary hypertension. Chest 129:1313-1321

8. Knirsch W, Eiselt M, Nurnberg J, Haas NA, Berger F, Dahnert I, Uhlemann F, Lange PE 2002 [Pulmonary plasma catecholamine levels and pulmonary hypertension in congenital heart disease]. Z Kardiol 91:1035-1043

9. Nootens M, Kaufmann E, Rector T, Toher C, Judd D, Francis GS, Rich S 1995 Neurohormonal activation in patients with right ventricular failure from pulmonary hypertension: relation to hemodynamic variables and endothelin levels. J Am Coll Cardiol 26:1581-1585

10. Voelkel MA, Wynne KM, Badesch DB, Groves BM, Voelkel NF 2000 Hyperuricemia in severe pulmonary hypertension. Chest 117:19-24

11. Nagaya N, Uematsu M, Satoh T, Kyotani S, Sakamaki F, Nakanishi N, Yamagishi M, Kunieda T, Miyatake K 1999 Serum uric acid levels correlate with the severity and the mortality of primary pulmonary hypertension. Am J Respir Crit Care Med 160:487-492

12. Davis GK, Bamforth F, Sarpal A, Dicke F, Rabi Y, Lyon ME 2006 B-type natriuretic peptide in pediatrics. Clin Biochem 39:600-605

13. Hammerer-Lercher A, Neubauer E, Muller S, Pachinger O, Puschendorf B, Mair J 2001 Head-to-head comparison of $\mathrm{N}$-terminal pro-brain natriuretic peptide, brain natriuretic peptide and $\mathrm{N}$-terminal pro-atrial natriuretic peptide in diagnosing left ventricular dysfunction. Clin Chim Acta 310:193-197

14. Masson S, Latini R, Anand IS, Vago T, Angelici L, Barlera S, Missov ED, Clerico A, Tognoni G, Cohn JN 2006 Direct comparison of B-type natriuretic peptide (BNP) and amino-terminal proBNP in a large population of patients with chronic and symptomatic heart failure: the valsartan heart failure (Val-HeFT) data. Clin Chem 52:1528-1538

15. Hobbs FD, Davis RC, Roalfe AK, Hare R, Davies MK, Kenkre JE 2002 Reliability of N-terminal pro-brain natriuretic peptide assay in diagnosis of heart failure: cohort study in representative and high risk community populations. BMJ 324:1498

16. Thomas JA, Marks BH 1978 Plasma norepinephrine in congestive heart failure Am J Cardiol 41:233-243

17. Cohn JN, Levine TB, Olivari MT, Garberg V, Lura D, Francis GS, Simon AB, Rector T 1984 Plasma norepinephrine as a guide to prognosis in patients with chronic congestive heart failure. N Engl J Med 311:819-823

18. Hoieggen A, Alderman MH, Kjeldsen SE, Julius S, Devereux RB, De Faire U, Fyhrquist F, Ibsen H, Kristianson K, Lederballe-Pedersen O, Lindholm LH, Nieminen MS, Omvik P, Oparil S, Wedel H, Chen C, Dahlof B 2004 The impact of serum uric acid on cardiovascular outcomes in the LIFE study. Kidney Int 65:1041-1049

19. Verdecchia P, Schillaci G, Reboldi G, Santeusanio F, Porcellati C, Brunetti P 2000 Relation between serum uric acid and risk of cardiovascular disease in essential hypertension. The PIUMA study. Hypertension 36:1072-1078

20. Freedman DS, Williamson DF, Gunter EW, Byers T 1995 Relation of serum uric acid to mortality and ischemic heart disease. The NHANES I epidemiologic follow-up study. Am J Epidemiol 141:637-644

21. Anker SD, Doehner W, Rauchhaus M, Sharma R, Francis D, Knosalla C, Davos CH, Cicoira M, Shamim W, Kemp M, Segal R, Osterziel KJ, Leyva F, Hetzer R, Ponikowski P, Coats AJ 2003 Uric acid and survival in chronic heart failure: validation and application in metabolic, functional, and hemodynamic staging. Circulation 107:1991-1997

22. Doehner W, Schoene N, Rauchhaus M, Leyva-Leon F, Pavitt DV, Reaveley DA, Schuler G, Coats AJ, Anker SD, Hambrecht R 2002 Effects of xanthine oxidase inhibition with allopurinol on endothelial function and peripheral blood flow in hyperuricemic patients with chronic heart failure: results from 2 placebo-controlled studies. Circulation 105:2619-2624

23. Chapman PJ, Bateman ED, Benatar SR 1990 Prognostic and therapeutic considerations in clinical primary pulmonary hypertension. Respir Med 84:489- 494 
24. Sandoval J, Bauerle O, Palomar A, Gomez A, Martinez-Guerra ML, Beltran M, Guerrero ML 1994 Survival in primary pulmonary hypertension. Validation of a prognostic equation. Circulation 89:1733-1744

25. Hoeper MM, Hohlfeld JM, Fabel H 1999 Hyperuricaemia in patients with right or left heart failure. Eur Respir J 13:682-685

26. Allanore Y, Borderie D, Meune C, Cabanes L, Weber S, Ekindjian OG, Kahan A 2003 N-terminal pro-brain natriuretic peptide as a diagnostic marker of early pulmonary artery hypertension in patients with systemic sclerosis and effects of calcium-channel blockers. Arthritis Rheum 48:3503-3508
27. Mukerjee D, Yap LB, Holmes AM, Nair D, Ayrton P, Black CM, Coghlan JG 2003 Significance of plasma N-terminal pro-brain natriuretic peptide in patients with systemic sclerosis-related pulmonary arterial hypertension. Respir Med 97:1230-1236

28. Souza R, Bogossian HB, Humbert M, Jardim C, Rabelo R, Amato MB, Carvalho CR 2005 N-terminal-pro-brain natriuretic peptide as a haemodynamic marker in idiopathic pulmonary arterial hypertension. Eur Respir J 25:509-513

29. Bendayan D, Shitrit D, Ygla M, Huerta M, Fink G, Kramer MR 2003 Hyperuricemia as a prognostic factor in pulmonary arterial hypertension. Respir Med 97:130-133

\section{Erratum}

In the article, "Upper Airway Dynamic Responses in Children with the Obstructive Sleep Apnea Syndrome," by Carol L Marcus, et al., appearing in Pediatric Research 2005; 57:99-107, Figures 2-5 did not reproduce adequately and are being reprinted in erratum for clarity.
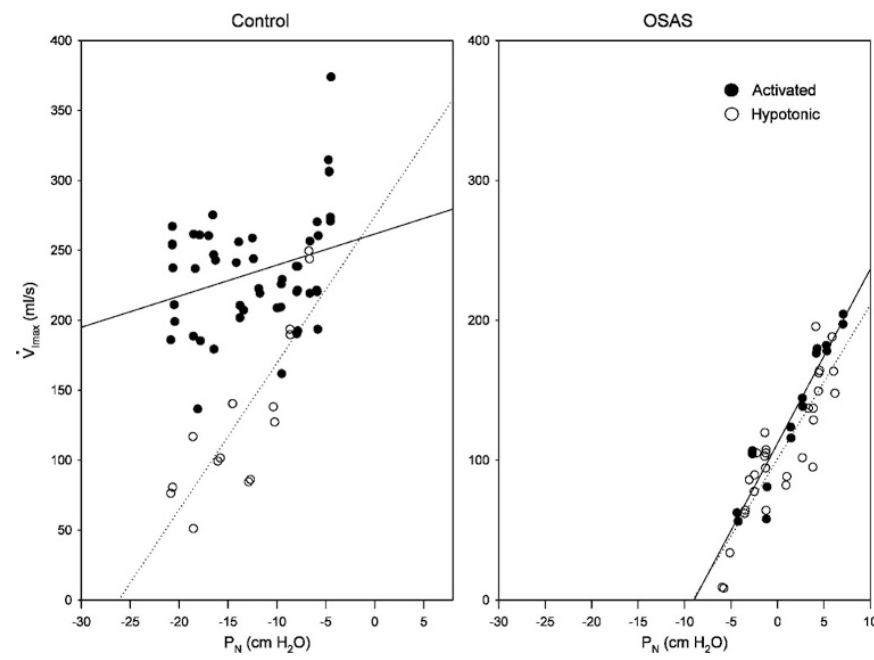

Fig. 2

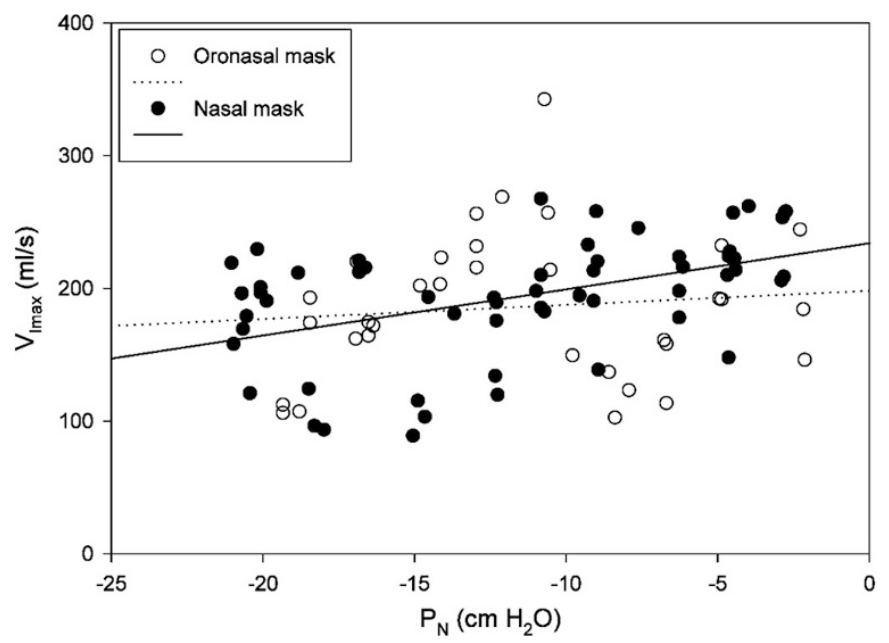

Fig. 4

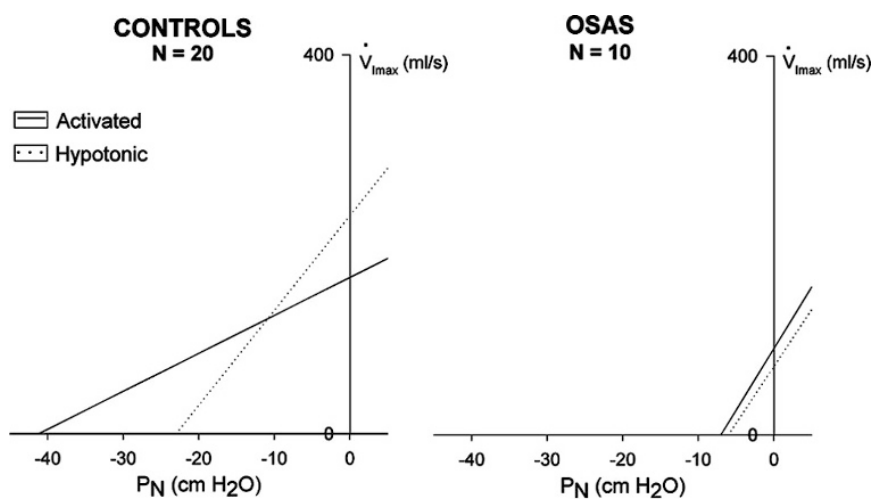

Fig. 3
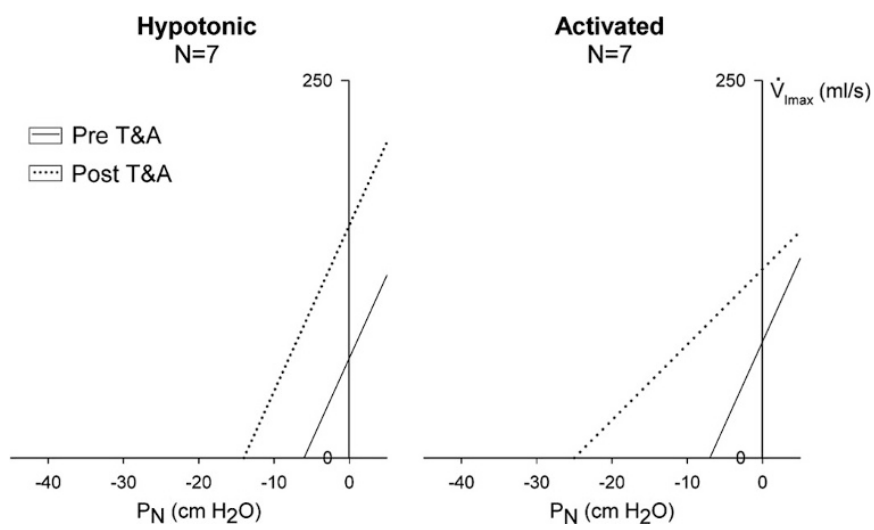

Fig. 5 